\title{
Fibromialgia: Discapacidad funcional, autoestima y perfil de personalidad
}

\author{
Maite Garaigordobil y Leila Govillard \\ Facultad de Psicología. Universidad del País Vasco.
}

\begin{abstract}
rosumen/ahstract:
El estudio tuvo como objetivo comparar el nivel de discapacidad funcional, dolor y agotamiento, el autoconcepto-autoestima, y grandes dimensiones de personalidad, en personas con y sin fibromialgia (FM). El estudio se realizó con 190 participantes, 140 afectados de FM y 50 no afectados, entre 28 y 75 años $(M=52,16$; $D T=9,18), 10$ varones y 180 mujeres. Se utilizaron 4 instrumentos de evaluación para medir las variables objeto de estudio: impacto de la fibromialgia, autoconcepto, autoestima y personalidad. Los resultados evidenciaron que las personas con FM, comparadas con las que no están afectadas por esta enfermedad, tienen significativamente un nivel superior de discapacidad funcional, dolor y agotamiento; un nivel inferior de autoestima; una capacidad superior de cooperación-empatía, de meticulosidad y amor por el orden, y un nivel inferior de control de emociones y de estabilidad emocional. En el resto de rasgos de personalidad (energía, afabilidad, tesón, apertura mental) no hubo diferencias significativas. Además, en las personas con FM no se han hallado indicios de sesgo o tendencia a ofrecer una imagen desfavorable, destacar dificultades, o presentar "conducta de enfermo".
\end{abstract}

The purpose of the study was to compare the level of functional impairment, pain and exhaustion, selfconcept-self-esteem, and major personality dimensions in people with and without fibromyalgia (FM). The study was carried out with 190 participants, 140 affected with FM and 50 not affected, between 28 and 75 years of age $(M=52.16, S D=9.18), 10$ males and 180 females. Four assessment instruments were administered to assess the target variables: impact of fibromyalgia, self-concept, self-esteem and personality. The results provided evidence that people with FM, compared with people who are not affected by this disease, have a significantly higher level of functional impairment, pain, and exhaustion, a lower level of self-esteem, a higher capacity of cooperativeness-empathy, scrupulousness, and a lower level of emotional control and emotional stability. In the remaining personality traits (energy, agreeableness, conscientiousness, mental openness), there were no significant differences. In addition, in people with FM, no signs of bias or tendency to present an unfavorable image, emphasize difficulties, or display "illness behavior" were found.

\section{palabras clave/keywords:}

Fibromialgia, autoestima, personalidad, discapacidad funcional.

Fibromyalgia, self-esteem, personality, functional impairment.

Agradecimientos:

Estudio financiado por el Departamento de Educación, Política Lingüistica y Educación del Gobierno Vasco (IT638-13), y por la Unidad de Formación e Investigación de la Universidad del País Vasco UPV/EHU (UFI PSIXXI 11/04). 


\section{Introducción}

La Fibromialgia (FM) se conoce desde hace más de 150 años con diferentes denominaciones como reumatismo muscular, fibrositis, neurastenia o incluso histeria. Fue Hench (1976) quién creó el término Fibromialgia, que posteriormente fue recogido por el American College of Rheumatology (ACR) (Wolfe et al., 1990) para establecer los criterios diagnósticos. En 1992 la Organización Mundial de la Salud (WHO) reconoce la FM, definida como una condición dolorosa, no articular, que envuelve los músculos, y es causa más común de dolor musculoesqueletal crónico y generalizado, habiendo sido incluida en su última revisión de la Clasificación Internacional de Enfermedades (CIE-10) con el código M79.9, dentro de las enfermedades reumatológicas, no psiquiátricas; perdurando hasta la actualidad los criterios diagnósticos establecidos por la ACR, que son la existencia de dolor generalizado durante al menos tres meses, hipersensibilidad al dolor en 11 o más de 18 localizaciones específicas o punto gatillo, y también el diagnóstico diferencial o descarte de otras patologías causantes (García-Campayo et al., 2010). Recientemente, Wolfe et al. (2010) han propuesto nuevas variables diagnósticas a tener en cuenta en la FM como la disfunción cognitiva, el sueño no reparador y múltiples síntomas somáticos como constatan estudios actuales (González-Ramírez, García-Campayo y Landero, 2011), siendo definida en la actualidad por algunos autores como un "síndrome de naturaleza funcional" en ausencia de biomarcadores fiables (Álvaro y Traver, 2010).

Los estudios que han analizado la discapacidad funcional en personas con FM han evidenciado niveles entre moderados y al- tos. En España, González-Ramírez et al. (2011) evidenciaron que las personas con FM presentan un grado de discapacidad alto obteniendo una puntuación media de 72,2 (rango 0-100) en una muestra de 165 pacientes. Este resultado es superior al encontrado por Mohamed, López y El Yousfi (2012) que con una muestra inferior $(\mathrm{N}=$ 80) obtienen una media de 60,90. En la misma línea, García-Bardón, Castel y Vidal (2006) encontraron que el 63\% de personas con FM presentaban un impacto moderado de la FM; y en Canadá, Iverson, Le Page, Koehler, Shojania y Badii (2007) hallaron que el $72 \%$ de personas con FM sufren un nivel alto de discapacidad funcional.

Son pocos los estudios que han explorado las conexiones entre autoconcepto-autoestima y FM. Blumer y Heilbronn (1981) han destacado que las personas con FM tienen baja autoestima y dependencia excesiva de la aceptación y el reconocimiento de los demás. Sin embargo, estudios actuales concluyen que el nivel de autoestima no es tan bajo en las personas afectadas, ya que obtienen una puntuación media de 28 (rango 10-40) (González-Ramírez et al., 2011; Mohamed et al., 2012). Por su parte, Morea, Friend y Bennett (2008), estudian la variable "autoconcepto de enfermo", señalando que influye significativamente en el afrontamiento de la FM.

Las conclusiones de la revisión realizada por Blumer y Heilbronn (1981) relacionan la FM con una personalidad perfeccionista, con amor por el orden, enfatizando la existencia de una tendencia de "sobrerealización" en los pacientes con esta enfermedad. Por otro lado, algunos investigadores han encontrado que las personas con FM, tienen una personalidad neurótica o emocionalmente poco estable, hallando me- 
diante instrumentos como el Inventario de Personalidad NEO-PI-R y el Inventario de Personalidad de Eysenck (EPQ), altas puntuaciones en neuroticismo en las personas con FM comparando con las personas sin FM (Besteiro et al., 2008; Malt, Olafsson, Lund, y Urson, 2002; Satalino, 2009), y con otros síndromes de dolor crónico, como la artritis reumatoide (Besterio et al., 2008). Sin embargo, el estudio de Zautra et al. (2005) refuta la asociación entre FM y neuroticismo o inestabilidad emocional, al no encontrarse diferencias significativas entre personas con FM y personas sin FM, y personas con artritis y reuma. En la misma línea, Ríos y Zautra (2011) comparando personas con FM, personas con osteoartritis y personas con ambos síndromes, concluyen que son estas últimas las que obtienen puntuaciones más elevadas en neuroticismo. Respecto a la distorsión de la percepción subjetiva o "conducta de enfermo", Besteiro et al. (2008), comparando una muestra de 60 personas con y sin FM, a través del Cuestionario de Orientación de Vida (OLQ), concluyen que las personas con FM tienen mayor distorsión subjetiva que las personas sin FM. La sobrevaloración de la enfermedad se ha considerado como una consecuencia del estado de ansiedad sensitiva o de la hipersensibilidad de las personas con FM, obteniendo un marcado perfil sómatico en comparación con personas sin FM y personas con dolor crónico (PérezPareja, 2010), o por el mantenimiento de un patrón de enfermo por beneficios secundarios (Ehrlich, 2003).

Con esta contextualización, el presente estudio tuvo como objetivo comparar a personas con y sin FM en relación a las siguientes variables: 1) nivel de discapacidad funcional, dolor y agotamiento; 2) autoconcepto-autoestima, y 3) rasgos o dimensiones de la personalidad (energía, afabilidad, tesón, estabilidad emocional, apertura mental). Con estos objetivos, y tomando como referencia los estudios previos, la presente investigación plantea 4 hipótesis: 1) Las personas con FM tendrán un nivel medio-alto de discapacidad funcional, dolor y agotamiento, y significativamente mayor que las personas sin FM; 2) Las personas con FM tendrán un nivel de autoconcepto y autoestima bajo y significativamente inferior que las personas sin FM; 3) Las personas afectadas por FM mostrarán similar perfil de personalidad que las que no tienen esta enfermedad, es decir, tendrán puntuaciones similares en grandes dimensiones de personalidad tales como energía, afabilidad, tesón, estabilidad emocional y apertura mental; y 4) Las personas con FM distorsionarán la imagen que tienen de sí mismas, en el sentido negativo (tendencia a dar una imagen desfavorable, destacar dificultades, o a presentar "conducta de enfermo").

\section{Método}

\section{Participantes}

La muestra está configurada por 190 participantes, 140 afectados de FM $(73,7 \%)$ y 50 no afectados $(26,3 \%)$, entre 28 y 75 años $(M=52,16 ; D T=9,18), 10$ son varones $(5,3 \%)$ y 180 mujeres $(94,7 \%)$. Los participantes con diagnóstico de FM pertenecen a la Asociación Guipuzcoana de Fibromialgia y Astenia Crónica, llamada Bizibide. Para la selección de la muestra de afectados de FM, se invitó a todas las personas de la asociación a participar en este estudio por correo y telefónicamente. El único requisito que se pidió fue que hubieran sido diagnosticados de FM, y que presentaran el justificante médico correspondiente. Del 
conjunto de los miembros de la asociación $(N=160)$, el 87,5\% participaron en el estudio $(n=140)$. Para seleccionar la muestra de no afectados, se tomó como referencia las características socio-demográficas básicas (sexo, edad, ocupación laboral) de las personas afectadas de FM, y se seleccionó una muestra espejo de 50 personas no afectadas con características socio-demográficas homogéneas. Uno de los criterios de selección muestral del grupo de personas no afectadas fue la ausencia de otras patologías a nivel físico o psíquico.

Las frecuencias (F) y porcentajes (\%) de participantes en función del sexo y de los 4 rangos de edad que contiene la muestra se presentan en la Tabla 1. Los participantes con FM $(n=140)$ tenían edades entre 28 y 75 años $(M=52,51 ; D T=8,94), 7$ son varones $(5 \%)$ y 133 mujeres (95\%). Los participantes sin FM $(n=50)$ tenían edades entre 29 y 75 años $(M=51,2 ; D T=9,83), 3$ son varones $(6 \%)$ y 47 mujeres $(94 \%)$. En la muestra de afectados por la FM (ver Tabla 1) las diferencias en función del sexo fueron significativas, $\chi^{2}=113,40, p<.001$, pero estas diferencias son habituales en los estudios llevados a cabo con personas con FM, en los que se constata un porcentaje

Tabla 1. Sexo y rangos de edad de los participantes con y $\sin \mathrm{FM}$

\begin{tabular}{|c|c|c|c|c|}
\hline \multirow{2}{*}{} & \multicolumn{2}{|c|}{ Con FM } & \multicolumn{2}{c|}{ Sin FM } \\
\cline { 2 - 5 } & $F$ & $\%$ & $F$ & $\%$ \\
\hline Varón & 7 & 5 & 3 & 6 \\
Mujer & 133 & 95 & 47 & 94 \\
\hline $28-38$ & 9 & 6,4 & 4 & 8 \\
$39-48$ & 36 & 25,7 & 13 & 26 \\
$49-59$ & 64 & 45,7 & 23 & 46 \\
$60-75$ & 30 & 21,4 & 9 & 18 \\
\hline
\end{tabular}

muy superior de mujeres frente a hombres con esta problemática. Entre los participantes con y sin FM, no se hallaron diferencias en función del sexo, $\chi^{2}=34,18, p>.05$, ni la edad, $\chi^{2}=0,074, p>.05$.

Además, se analizó si existían diferencias en la ocupación laboral de los participantes con y sin FM. Los resultados mostraron que de los afectados de FM un 19,5\% actualmente no tienen una ocupación laboral, están de baja, jubilados o incapacitados; un $16,4 \%$ son amas de casa, y un $64,1 \%$ realizan distintas profesiones. En la muestra de los no afectados, un 9\%, actualmente no tienen una ocupación laboral, un 13,6\% son amas de casa, y un $77,4 \%$ realizan distintas profesiones. Como se puede observar en la muestra de afectados hay un porcentaje significativamente más alto de personas sin ocupación laboral, situación que se explica desde el impacto de la enfermedad $\left(\chi^{2}=\right.$ 26,33, $p<.05$ ).

\section{Instrumentos de Evaluación}

Para evaluar las variables objeto de estudio se administraron 4 instrumentos de evaluación con garantías de fiabilidad y validez.

FIQ. Cuestionario de Impacto de la Fibromialgia (Burckhardt, Clark y Bennet, 1994, adapt. Monterde, Salvat, Montull y Fernández-Ballart, 2004). Mide el impacto de la fibromialgia en la capacidad funcional y en la calidad de vida de las personas que la padecen. Evalúa el impacto de la FM en la capacidad física, en la posibilidad de realizar el trabajo habitual, es decir, el grado en que la FM afecta a la actividad habitual, síntomas físicos (dolor, fatiga, sensación de cansancio y rigidez) y el estado emocional (ansiedad y depresión). Es un cuestionario multidimensional que mediante preguntas que hacen referencia a la semana anterior 
a la realización del test, valora los componentes del estado de salud que suelen estar más afectados en pacientes con FM. Consta de 10 ítems que permiten valorar la capacidad funcional del paciente mediante preguntas acerca de actividades relacionadas con la vida diaria (comprar, conducir, hacer la colada, etc.), y valorar el grado de dolor y agotamiento. En la adaptación española el valor del coeficiente de correlación intraclase total fue de .81. El S-FIQ mantuvo las propiedades de la versión original y se confirmó su adecuación como herramienta de medida en pacientes españoles con FM.

LAEA. Listado de adjetivos para la evaluación del autoconcepto (Garaigordobil, 2011). El listado está compuesto por 57 adjetivos y se solicita al sujeto que valore en una escala de estimación de 0 a 4 (nada-mucho) en qué grado estos adjetivos le definen o describen su personalidad. Un estudio realizado con una muestra de 634 sujetos obtuvo un alpha de Cronbach de .92, y un coeficiente de Spearman-Brown de .83 (Garaigorodobil, Pérez y Mozaz, 2008). La fiabilidad test-retest obtenida con una muestra de 142 estudiantes universitarios y un intervalo de 40 días fue alta $(r=$ .83), evidenciando su estabilidad temporal. Para analizar la validez del LAEA, se realizaron correlaciones con otros instrumentos que miden el autoconcepto (AF-5, García y Musitu, 1999) obteniéndose correlaciones significativas positivas $(r=.71)$ que muestran una validez de constructo aceptable. Estudios de validez discriminante del LAEA con una muestra de 1.578 sujetos de 12 a 65 han confirmado las relaciones negativas entre el LAEA y síntomas psicopatológicos, y relaciones positivas con empatía, con inteligencia emocional, con rasgos de personalidad como amabilidad y responsabilidad (Garaigordobil, 2011).
RSE. Escala de autoestima (Rosenberg, 1965). Evalúa la autoestima general con 10 afirmaciones que aluden a sentimientos globales de autovaloración (en general estoy satisfecho conmigo mismo), 5 de los cuales están redactados en sentido positivo y 5 en sentido negativo. El sujeto debe leer las afirmaciones e informar en qué medida pueden ser aplicadas a él mismo, haciendo la valoración sobre una escala de tipo Likert con 4 categorías de respuesta (desde muy de acuerdo, a muy en desacuerdo). La fiabilidad de la prueba ha sido ampliamente documentada en la literatura. McCarthy y Hoge (1982) han informado de coeficientes de consistencia (alpha de Cronbach) que se sitúan entre .74 y .77 , y de fiabilidad testretest de .63 (intervalo de 7 meses) y de .85 (intervalo de 2 semanas). La validez de la escala como medida unidimensional de la autoestima ha sido también comprobada en varios estudios (Garaigorodobil et al., 2008; Rosenberg, 1965; Silber y Tippett, 1965).

BFQ. Cuestionario de los Cinco Grandes Factores (Caprara, Barbaranelli, y Borgogni, 1993). Evalúa 5 grandes factores o dimensiones de la personalidad y 10 subdimensiones implícitas en estos: 1) Energía (dinamismo, dominancia); 2) Afabilidad (cooperación-empatía, cordialidad-amabilidad); 3) Tesón (perseverancia, escrupulosidad); 4) Estabilidad Emocional (control de emociones, control de impulsos); y 5) Apertura mental (apertura a la cultura, apertura a la experiencia). Contiene 132 afirmaciones, y la persona debe informar en qué medida pueden aplicarse a sí misma los contenidos de las mismas, utilizando una escala de Likert de 5 puntos (1 completamente falso para mi - 5 completamente verdadero para mí). Los resultados de estudios 
psicométricos con la adaptación española del BFQ y estudios transculturales evidencian su fiabilidad y validez. Con la muestra española los coeficientes alpha fueron adecuados (energía .75, afabilidad .73, tesón .79 , estabilidad emocional .87, apertura mental .76). En la validez interna, los estudios que analizan la estructura factorial evidencian altos índices de congruencia en el contraste con las soluciones factoriales aisladas en grupos cultural y lingüísticamente distintos. Los factores extraídos coinciden significativamente con las escalas definidas teóricamente (Caprara et al., 1993).

\section{Diseño y Procedimiento}

Después de seleccionar la muestra, se procedió a la administración de las pruebas a las 140 personas afectadas de FM. La duración de evaluación fue de 2 horas aproximadamente, realizando varios descansos en el proceso de la misma. Los participantes con FM, fueron distribuidos al azar en grupos de $10-15$ personas, y los miembros del equipo investigador entregaron los cuestionarios estando a disposición de los participantes para resolver las dudas que pudieran surgir en la cumplimentación de los mismos. Posteriormente, tras seleccionar a la muestra espejo, se realizó la administra- ción a 50 personas no afectadas de FM, con el mismo procedimiento de evaluación. El estudio cumplió los valores éticos requeridos en la investigación con seres humanos respetando los principios fundamentales incluidos en la Declaración de Helsinki, en sus actualizaciones, y en las normativas vigentes (consentimiento informado y derecho a la información, protección de datos personales y garantías de confidencialidad, no discriminación, gratuidad y posibilidad de abandonar el estudio en cualquiera de sus fases). El análisis de datos se realizó con el paquete estadístico SPSS 18.0.

\section{Resultados}

\section{Discapacidad funcional en participantes con y sin FM}

Para explorar si existen diferencias significativas en discapacidad funcional, dolor y agotamiento, entre los participantes con y sin FM, en primer lugar, se realizó un análisis de varianza multivariado (MANOVA), con las puntuaciones obtenidas en todas las variables del FIQ. Los resultados del MANOVA, Lambda de Wilks, $\Lambda=.280$, indicaron la existencia de diferencias estadísticamente significativas entre ambas condiciones, $F(3,185)=158,86, p<.001$, siendo la magnitud del efecto grande $\left(\eta^{2}=\right.$

Tabla 2. Media, desviación típica y análisis de varianza en discapacidad funcional, dolor y agotamiento de los participantes con y $\sin \mathrm{FM}$

\begin{tabular}{|c|c|c|c|c|c|}
\hline & \multicolumn{2}{|c|}{$\begin{array}{c}\text { Participantes con FM } \\
(n=140)\end{array}$} & \multicolumn{2}{c|}{$\begin{array}{c}\text { Participantes sin FM } \\
(n=50)\end{array}$} & \multirow{2}{*}{$F(1,188)$} \\
\cline { 2 - 5 } & $\mathrm{M}$ & $\mathrm{DT}$ & $\mathrm{M}$ & $\mathrm{DT}$ & \\
\hline Grado discapacidad funcional & 74,34 & 13,81 & 25,54 & 16,13 & $414,41^{* * *}$ \\
\hline Grado de dolor & 8,11 & 1,69 & 2,29 & 1,81 & $412,66^{* * *}$ \\
\hline Grado de agotamiento & 8,72 & 1,65 & 4,14 & 2,43 & $214,24^{* * *}$ \\
\hline
\end{tabular}

${ }^{* * *} p<.001$ 
$.720, r=.84)$. Tras comprobar los supuestos básicos (normalidad, homocedasticidad...), se procedió a realizar un análisis de varianza del grado de discapacidad funcional, de dolor y de agotamiento de los participantes con y sin FM, cuyos resultados se presentan en la Tabla 2.

Los resultados (ver Tabla 2) permiten observar una puntuación media significativamente superior en el grado de discapacidad funcional en los participantes con FM ( $M=$ $74,34)$, versus sin FM $(M=25,54)$ (rango de puntuación 0-100). Respecto al grado de dolor, también se observa una puntuación media significativamente superior en las personas afectadas con FM $(M=8,11)$, frente a los que no tienen esta enfermedad $(M=2,29)$ (rango de puntuación 0-10). Y en la misma dirección apuntan los resultados sobre el grado de agotamiento en el que también se evidencia una puntuación media significativamente superior en los participantes con FM $(M=8,72)$, frente a los participantes sin FM $(M=4,14)$ (rango de puntuación 0-10).

\section{Autoconcepto y autoestima en participantes con y sin FM}

Con la finalidad de comparar el nivel del autoconcepto y la autoestima en las personas con y sin FM se realizan análisis des- criptivos y de varianza (ANOVA) con los datos obtenidos con el LAEA y el RSE, cuyos resultados se presentan en la Tabla 3.

Tal y como se puede observar (ver Tabla $3)$, las puntuaciones en el autoconcepto de los participantes con FM $(M=150,08)$, y $\sin$ FM $(M=146,44)$ son similares. Sin embargo, en autoestima se encuentran puntuaciones significativamente inferiores en los participantes con FM $(M=29,06)$, frente a los que no tienen esta enfermedad $(M=$ $30,86)$. No obstante, teniendo en cuenta los baremos elaborados con muestra española en ambas pruebas (Garaigordobil, Pérez, y Mozaz, 2008), el nivel de autoconcepto en ambas condiciones está dentro del promedio, mientras que el nivel de autoestima de los participantes con FM es ligeramente bajo (percentil 25), y el de los participantes sin FM está dentro del promedio.

\section{Rasgos de personalidad en participantes con y sin FM}

Para explorar si existe un perfil de personalidad específico de las personas con FM, se analizó si existen diferencias significativas en rasgos o dimensiones de la personalidad entre los participantes con y $\sin$ FM, en primer lugar, se realizó un análisis de varianza multivariado (MANOVA), con las puntuaciones obtenidas en todas las variables del

Tabla 3. Media, desviación típica y análisis de varianza en autoconcepto y autoestima en participantes con y sin FM

\begin{tabular}{|c|c|c|c|c|c|}
\hline & \multicolumn{2}{|c|}{$\begin{array}{c}\text { Participantes con FM } \\
(n=140)\end{array}$} & \multicolumn{2}{c|}{$\begin{array}{c}\text { Participantes sin FM } \\
(n=50)\end{array}$} & \multirow{2}{*}{$F(1,188)$} \\
\cline { 2 - 5 } & $M$ & $D T$ & $M$ & $D T$ & \\
\hline Autoconcepto & 150,08 & 27,70 & 146,44 & 21,69 & 0,70 \\
\hline Autoestima & 29,06 & 4,52 & 30,86 & 3,64 & $6,37^{*}$ \\
\hline
\end{tabular}

${ }^{*} p<.05$ 
BFQ analizadas globalmente. Los resultados del MANOVA, Lambda de Wilks, $\Lambda$ = .770 , indicaron la existencia de diferencias estadísticamente significativas entre ambas condiciones, $F(16,173)=3,23, p<.001$, siendo la magnitud del efecto media $\left(\eta^{2}=\right.$ $.230, r=.47)$. Posteriormente, se realizaron análisis descriptivos y univariantes cuyos resultados se presentan en la Tabla 4.

Los resultados de los análisis de varianza (ver Tabla 4), evidencian diferencias significativas entre los participantes con y sin FM en 4 variables de personalidad. En cooperación-empatía (comprensión de los problemas y necesidades de los demás y la cooperación con ellos), se observa una puntuación significativamente superior en participantes con FM $(M=45,58)$ versus $\sin$ FM $(M=43,8)$. En escrupulosidad (meticulosidad, amor por el orden), también se evidencia una puntuación significativamente superior en los afectados por FM $(M=41,89)$, frente a los participantes sin FM $(M=39,00)$. Sin embargo, en control de las emociones (control de los estados de tensión asociados a experiencias emotivas), se observa una puntuación significativamente inferior en las personas con FM $(M=$ $27,61)$, frente a las que no tienen FM $(M=$ 34,2). Y en el factor estabilidad emocional

Tabla 4. Media, desviación típica y análisis de varianza en rasgos de personalidad en participantes con y sin FM

\begin{tabular}{|c|c|c|c|c|c|}
\hline & \multicolumn{2}{|c|}{$\begin{array}{l}\text { Participantes con FM } \\
\qquad(n=140)\end{array}$} & \multicolumn{2}{|c|}{$\begin{array}{l}\text { Participantes sin FM } \\
\qquad(n=50)\end{array}$} & \multirow[t]{2}{*}{$F(1,188)$} \\
\hline & $M$ & $D T$ & $M$ & $D T$ & \\
\hline Dinamismo & 38,85 & 7,27 & 38,38 & 6,36 & 0,164 \\
\hline Dominancia & 31,86 & 5,50 & 30,90 & 5,12 & 1,172 \\
\hline Energía & 70,95 & 9,22 & 69,28 & 9,22 & 1,208 \\
\hline Cooperación-Empatía & 45,58 & 5,37 & 43,8 & 4,36 & $4,42 *$ \\
\hline Cordialidad & 40,74 & 5,89 & 40,04 & 6,89 & 0,47 \\
\hline Afabilidad & 86,46 & 9,01 & 83,96 & 10,39 & 2,61 \\
\hline Escrupulosidad & 41,89 & 6,88 & 39,00 & 8,22 & $5,82 *$ \\
\hline Perseverancia & 40,96 & 6,86 & 40,56 & 6,26 & 0,13 \\
\hline Tesón & 82,79 & 11,09 & 80,08 & 11,32 & 2,16 \\
\hline Control Emociones & 27,61 & 7,13 & 34,2 & 7,07 & $31,54 * * *$ \\
\hline Control Impulsos & 29,75 & 7,87 & 32,22 & 7,37 & 3,74 \\
\hline Estabilidad emocional & 57,5 & 13,72 & 66,42 & 13,21 & $15,85^{* * *}$ \\
\hline Apertura cultura & 37,84 & 6,43 & 38,92 & 7,16 & 0,98 \\
\hline Apertura experiencia & 38,71 & 5,94 & 38,20 & 5,57 & 0,27 \\
\hline Apertura mental & 76,42 & 10,57 & 76,98 & 10,34 & 0,10 \\
\hline Distorsión & 30,10 & 6,95 & 28,52 & 5,42 & 2,11 \\
\hline
\end{tabular}

${ }^{* * *} p<.001,{ }^{* *} p<.01,{ }^{*} p<.05$ 
(ansiedad, vulnerabilidad, emotividad e impulsividad), se constata una puntuación significativamente inferior en los participantes con FM $(M=57,5)$, frente a los participantes $\sin$ FM $(M=66,42)$. En el resto de los rasgos no se observan diferencias estadísticamente significativas. Excepto en control de emociones que la puntuación es baja, en el resto de las variables de personalidad las puntuaciones de las personas con FM están dentro del promedio.

Por otro lado, en la escala de Distorsión, que valora la tendencia a ofrecer un perfil falseado o sesgado de uno mismo, en sentido positivo (tendencia a ofrecer una imagen positiva y a negar las limitaciones), y en sentido negativo (tendencia a dar una imagen desfavorable, destacar dificultades, o a presentar "conducta de enfermo"), no se encontraron diferencias significativas entre ambas condiciones (con y sin FM).

\section{Discusión}

El estudio tuvo como objetivo comparar el nivel de discapacidad funcional, de dolor y agotamiento, el autoconcepto-autoestima, y diversos rasgos o dimensiones de personalidad en personas con y sin FM. En primer lugar, los resultados han evidenciado que las personas con FM tienen un nivel significativamente superior de discapacidad funcional, de dolor y de agotamiento respecto a las personas que no padecen esta enfermedad, lo que ratifica la hipótesis 1 . Los datos ponen de relieve un alto nivel de discapacidad funcional para desarrollar las actividades cotidianas, así como un alto grado de dolor y de agotamiento. Estos resultados confirman los obtenidos en otros trabajos (González-Ramírez et al., 2011; Mohamed et al., 2012) que han evidenciado que las personas con FM presentan un grado de discapacidad entre moderadointenso y alto (entre 59 y 80 sobre 100). En relación al grado de dolor, los resultados de nuestro estudio muestran puntuaciones algo superiores $(M=8,11)$ que los evidenciados en el trabajo de Collado et al. (2001) que hallaron una puntuación media de 7,4. $\mathrm{Y}$ respecto al agotamiento, Collado et al. (2001) obtienen un nivel medio de 6,3, también algo inferior al obtenido en nuestro trabajo $(M=8,72)$. El hecho de que todos los estudios revisados confirmen el carácter discapacitante de esta enfermedad, contrasta con el hecho de que en España y otros muchos países no esté reconocida como tal, existiendo un desamparo legal al respecto, y haciendo necesaria la figura del psicólogo en el ámbito legal, en concreto en la valoración del impacto de la enfermedad en la calidad de vida de las personas afectadas.

En segundo lugar, los resultados han evidenciado que las personas con FM comparadas con las que no tienen esta enfermedad tienen un nivel similar de autoconcepto. Sin embargo, las personas con FM muestran significativamente un nivel ligeramente bajo de autoestima e inferior que los que no tienen esta enfermedad, por lo que la hipótesis 2 no se confirma completamente, ya que se había hipotetizado que manifestarían también bajo autoconcepto. Por lo tanto, en la autovaloración cognitiva (autoconcepto) las personas con y sin FM muestran similar autoimagen, sin embargo, en una autovaloración más afectiva (autoestima) muestran un nivel inferior respecto a los que no padecen esta enfermedad, lo que implica una afectación emocional que podría estar relacionada con los niveles inferiores de estabilidad y control emocional observados en el BFQ. Estos resultados apuntan en la misma dirección que los obtenidos por González- 
Ramírez et al. (2011), y Mohamed et al. (2012) que hallaron un nivel bajo-moderado de autoestima en las personas con FM. Sin embargo, contradicen lo planteado por Blumer y Heilbronn (1981) que subrayan la existencia de un nivel bastante bajo de autoestima. Quizás las discrepancias pueden ser explicadas por el hecho de que el trabajo de Blumer y Heilbronn (1981) es una revisión de estudios, en los que no todos comparan la autoestima de las personas con y sin FM, o por la heterogeneidad de los instrumentos de evaluación administrados que dificulta comparar los resultados.

En tercer lugar, los resultados han puesto de relieve que las personas con FM, comparadas con las que no tienen FM, tienen significativamente una capacidad superior de cooperación-empatía (comprensión de los problemas y necesidades de los demás, conductas de cooperación), y de escrupulosidad (meticulosidad, amor por el orden), mientras que muestran un nivel significativamente inferior de control de emociones (control de los estados de tensión asociados a experiencias emotivas), y de estabilidad emocional (control de la ansiedad, la emotividad y la impulsividad). No obstante, hay que destacar que teniendo en cuenta los baremos de la prueba, excepto en control de emociones, las puntuaciones en estos rasgos están dentro del promedio, no constituyendo las diferencias encontradas entre participantes con y sin FM, indicadores de morbilidad en la personalidad de los afectados con FM. En el resto de rasgos de personalidad no se hallan diferencias significativas, apreciándose en general un perfil similar en los participantes con y sin FM. Por consiguiente, la hipótesis 3 se confirma casi en su totalidad, ya que las personas con y $\sin$ FM muestran similar nivel de energía, afabilidad, tesón, y apertura mental. Únicamente, en contra de lo hipotetizado, las personas con FM han mostrado bajo nivel de control emocional.

Gran parte de la literatura relaciona la FM con una personalidad perfeccionista, ordenada (Blumer y Heilbronn, 1981) y los resultados obtenidos en nuestro estudio confirman que las personas con FM significativamente son más meticulosas y les gusta el orden. Además, los resultados del estudio apuntan en la misma dirección que otras investigaciones que han hallado menor estabilidad emocional en las personas con FM (Besteiro et al., 2008; Malt et al., 2002). No obstante, en el presente estudio la puntuación en estabilidad emocional, aunque inferior a las personas sin FM, está dentro del promedio, por lo que no cobra relevancia como factor disfuncional en la FM, lo que coincide con los hallazgos del estudio de Zautra et al. (2005). A pesar de las diferencias encontradas, cabe destacar que las tendencias son similares en ambos grupos, no apreciándose un perfil de personalidad diferencial entre ambos, lo que implica el descarte de una personalidad mórbida como etiología de esta enfermedad, y la prudencia a la hora de catalogar a las personas afectadas como enfermos psiquiátricos, y centrar el tratamiento en el afrontamiento de la FM, utilizando todos los recursos positivos que conservan muchas de las personas afectadas.

Finalmente, los resultados no han evidenciado en las personas con FM indicios de sesgo o tendencia a ofrecer un perfil falseado de sí mismas, en sentido negativo (tendencia a dar una imagen desfavorable, destacar dificultades, o presentar "conducta de enfermo"), lo que refuta la hipótesis 4. Respecto a la existencia de sesgo o ten- 
dencia a ofrecer un perfil falseado, como enfermo, algunos autores (Besteiro et al., 2008; Ehrlich, 2003; Pérez-Pareja 2010) postulan la presencia de puntuaciones más altas en distorsión de la percepción subjetiva, o "conducta de enfermo" en las personas con FM; sin embargo, los resultados de nuestro estudio no evidencian indicios de distorsión en las personas con FM. En consecuencia, la hipótesis de la conducta de enfermo o del beneficio secundario no se sustenta como fenómeno característico en las personas con FM.

Quizás las discrepancias en los resultados pueden deberse a los distintos instrumentos de evaluación utilizados en las diferentes investigaciones (BFQ, MMPI, NEO...), o a la diferencial interpretación de los resultados, ya que las puntuaciones que se han obtenido en la mayor parte de los rasgos de personalidad de los participantes con y sin FM están dentro del promedio; por lo tanto, en contra de lo postulado por Blumer y Heilbronn (1981), se descarta la existencia de un patrón de personalidad proclive al dolor en las personas con FM, aunque algunos rasgos que se hallan más acentuados en éstas (por ejemplo, escrupulosidad), si pueden incidir en la evolución y afrontamiento de la enfermedad que padecen, pero no constituyen su etiología. Respecto a la conducta de enfermo por beneficios secundarios económicos, si bien es cierto que un porcentaje inferior al $45 \%$ de personas afectadas reciben algún tipo de ayuda o prestación debido a la enfermedad, el balance total de su economía, teniendo en cuenta los gastos derivados de su estado, resulta bastante negativo para la mayor parte de los afectados. Así, el 81,4\% de las personas con FM refiere que su economía y nivel de vida ha empeorado, o que han su- frido dificultades económicas a raíz de padecer la FM debido a los gastos derivados de los fármacos, los tratamientos recibidos, y por no poder mantener su actividad laboral, lo que lejos de suponer un beneficio económico, supone un menoscabo en la vida de los afectados, siendo la hipótesis del beneficio secundario poco factible en la mayor parte de los casos estudiados.

El estudio aporta información proveniente de la comparación de datos de las personas afectadas con un grupo normativo o de personas sin FM, aspecto ausente en muchos estudios previos. Entre las principales aportaciones e implicaciones del estudio cabe destacar el haber ratificado el carácter incapacitante de la FM, que contrasta con la falta de su reconocimiento, lo que sitúa a las personas afectadas en una situación de desamparo legal y social, y hace necesaria la figura del psicólogo tanto en la divulgación de la enfermedad, como en la prevención de la victimización de las personas afectadas. Además, se ha encontrado una afectación emocional en las personas con FM, que presentan menor autoestima y bajo control de emociones respecto a las personas no afectadas, lo que implica la necesidad de tratar desde un enfoque multidisciplinar a las personas afectadas, incluyendo la intervención psicológica.

Entre las limitaciones del estudio cabe destacar: 1) la escasez de varones en la muestra que impide controlar determinadas variables sociodemográficas fundamentales en el estudio de la personalidad, como el sexo y la edad; 2) la diferencia entre el tamaño de la muestra con y sin FM, lo que sugiere matizar las conclusiones extraídas; 3) la naturaleza transversal del estudio que no permite conocer la evolución de las personas afectadas, tanto en su capacidad fun- 
cional, como en su personalidad a través del tiempo, y a su vez en relación a otros síndromes de dolor crónico; y 4) el uso de autoinformes con el sesgo de deseabilidad que implican.

Como líneas futuras de investigación se proponen: 1) la realización de estudios longitudinales para conocer la evolución de las personas afectadas, 2) la comparación de los resultados obtenidos con una muestra de personas afectadas por otros síndromes de dolor crónico; y 3) la aplicación y evaluación de tratamientos dirigidos a mejorar el afrontamiento y el impacto de la enfermedad, utilizando enfoques multidisciplinares que aborden tanto aspectos médicos, como psicológicos, actividad física, ocupacional, etc.

\section{Referencias}

Álvaro, T., y Traver, F. (2010). Una visión psiconeuroinmunológica de la fibromialgia. Revista de Psicopatología y Psicología Clínica, 15(3), 149-163.

Besteiro, J., Álvarez, M., Lemos, S., Muñiz, J., Costas, C., y Weruaga, A. (2008). Personality dimensions, sense of coherente and perceived health status in patients with fibromyalgia syndrome. International Journal of Clinical and Health Psychology, 8(2), 411-427.

Blumer, D., y Heilbronn, M. (1981). The pain-prone disorder: A clinical and psychological profile. Psychosomatics, 22, 395-397.

Burckhardt, C., Clark, S., y Bennet, R. (1991). The Fibromyalgia Impact Questionnaire: development and validation. Journal of Rheumatology, 18, 728-733.

Caprara, G.V., Barbaranelli, C., y Borgogni, L. (1993). BFO. Cuestionario de los Cinco Grandes Factores. Madrid: TEA. (adaptación española de José Bermudez)

Collado, A., Torres, X., Arias, A., Cerdá, D., Vilarrasa, R., Valdés, M., y Muñoz, J. (2001). Eficacia del tratamiento multidisciplinario del dolor crónico incapacitante del aparato locomotor. Medicina Clínica, 117, 401-405.
Ehrlich, G.E. (2003). Pain is real, Fibromialgia isn't. The Journal of Rheumatology, 30, 1666-1667.

Garaigordobil, M. (2011). LAEA. Listado de adjetivos para la evaluación del autoconcepto en adolescentes y adultos. Madrid: TEA.

Garaigordobil, M., Pérez, J. I., y Mozaz, M. (2008). Selfconcept, self-esteem and psychopathological symptoms. Psicothema, 20(1), 114-123.

García, F., y Musitu, G. (1999). AF-5. Autoconcepto Forma 5. Madrid: TEA.

García-Bardón, V., Castel, B., y Vidal, J. (2006). Evidencia científica de los aspectos psicológicos en la fibromialgia. Posibilidades de intervención. Reumatología Clínica, 2(1), 38-43.

García-Campayo, J., Alegre, C., Tomás, M., Gómez Argüelles, J. M., Blanco, E., Gobbo, M., Pérez Martín, A., Martínez Salio, A., Vidal, J., Altarriba, E., y Gómez de la Cámara, A. (2010). Documento de Consenso interdisciplinar para el tratamiento de la fibromialgia. Actas Españolas de Psiquiatría, 38(2), 108-120.

González-Ramírez, M., García-Campayo, J., y Landero, R. (2011). El papel de la teoría transaccional del estrés en el desarrollo de la fibromialgia: un modelo de ecuaciones estructurales. Actas Españolas de Psiquiatría, 39(2), 81-7.

Hench, P. K. (1976). Nonarticular rheumatism, twentysecond rheumatism review: Review of the American and English literature for the years 1973 and 1974. Arthritis and Rheumatism, 19, 1081-1089.

Iverson, G. L., Page, J. L., Koehler, B. E., Shojania, K., y Badii, M. (2007). Test of memory malingering (TOMM) scores are not affected by chronic pain or depression in patients with fibromyalgia. Clinical Neurophsychology, 21(3), 532-546.

Malt, E. A., Olafsson, S., Lund, A., y Urson, H. (2002). Factors explaining variance in perceived pain in women with fibromyalgia. BMC Musculoskeletal Disorders, 3, 12.

McCarthy, J. D., y Hoge, D. R. (1982). Analysis of age effects in longitudinal study of adolescent self-esteem. $D e$ velopmental Psychology, 18, 372-379.

Mohamed, K., López, C .J., y El Yousfi, M. (2012). Protocolo de Evaluación Psicosocial para Enfermas de Fibromialgia. Trances, 4(2), 2331-246.

Monterde, S., Salvat, I., Montull, S., y Fernández-Ballart, J. (2004). Validación de la versión Española del Fibromialgia Impact Questionnaire. Revista Española de Reumatología, 31(9), 507-513.

Morea, J., Friend, R., y Bennett, R. (2008). Conceptualizing and measuring illness self-concept: A comparison with self-esteem and optimism in predicting fibromyalgia adjustment. Research in Nursing and Health, 31(6), 563-575. 
Pérez-Pareja, J., Sese, A., Gonzalez-Ordi, H., y Palmer, A. (2010). Fibromialgy and chronic pain: Are there discriminating patterns by using the Minnesota Multiphasic Personality Inventory-2 (MMPI-2)? International Journal of Clinical and Health Psychology, 10 (1), 41-56.

Ríos, R., y Zautra, A. (2011). Socioeconomic Disparities in Pain: The Role of Economic Hardship and Daily Financial Worry. Health Psychology, 30(1), 58-66.

Rosenberg, M. (1965). Society and the adolescent selfimage. Princeton, NJ: Princeton University Press.

Satalino, A. (2009). Personality traits, perceived stress, and coping styles in patients with chrocic lyme disease and fibromyalgia. Dissertation Abstracts International: Section B: The Sciences and Engineering, 69(11-B), 7149.

Silber, E., y Tippett, J. (1965). Self-esteem: clinical assessment and measurement validation. Psychological Reports, 16, 1017-1071.

WHO. World Health Organization (1992). Internacional Statistical Classification of Diseases and Related Problems. ICD-10. Geneve: Author. [Organización Mundial de la Salud OMS (2002). CIE-10. Décima Revisión de la Clasificación Internacional de las Enfermedades y Problemas Relacionados con la Salud. Madrid: Meditor]

Wolfe, F., Clauw, D.J., Fitzcharles, M.A., Goldenberg, D.L, Katz, R.S., y Mease, P. (2010). The American collage of rheumatology preliminary diagnostic criteria for fibromyalgia and measurement of syptom severity. Arthritis Care \& and Research, 62,600-610.

Wolfe, F., Smythe, H. A., Yunus, M. B., Bennet, R. M., Bombardier, C., Goldenberg, D. L., Tugwel, P., Campbell, S. M., Abeles, M., y Clark, P. (1990). The American College of Rheumatology criterial for the classification of fibromyalgia. Report of the Multicenter Criteria Committee. Arthritis Rheumatology, 33, 160-172.

Zautra, A., Fasman, R., Reich, J., Harakas, P., Johnson, L., Olmsted, M., y Davis, M. (2005). Fibromyalgia: evidence for deficits in positive affect regulation. Psychosomatic Medicine, 67(1), 147-155.

Fecha de recepción: 12/04/2013

Fecha de aceptación: 25/11/2013 